\title{
La qualification dans la sociologie française : en quête des femmes.
}

\author{
Anne-Marie DAUNE-RICHARD
}

\author{
Paru in Laufer J., Marry C. et Maruani M; (eds), Le travail du genre. Les sciences \\ sociales du travail à l'épreuve des différences de sexe. La découverte, 2003. P. 138-150
}

L'organisation du travail industriel qui se met en place en France dès les années vingt repose sur une division taylorienne très poussée entre du travail d'exécution et un encadrement chargé de la conception et de la mise en œuvre des méthodes. Dans cette organisation scientifique du travail -à l'opposé de celle des métiers- le procès de travail est découpé et pré défini : le poste de travail devient l'unité de base.

Est-ce l'essor particulier de cette division du travail en France qui y a marqué le débat sur la qualification? Quoiqu'il en soit, ce débat s'est construit dans la sociologie française des années 1950-1960 sur la question de savoir ce qui est en jeu dans l'opération de qualification : le poste de travail et les aptitudes qu'il requiert ou le travailleur et les qualités qu'il possède?

M. Campinos-Dubernet et C. Marry (1986) ont analysé les fondements et les évolutions des débats français -tant sociologiques qu'économiques- des années 50-70 autour de la qualification. Elles opposent une "conception substantialiste", préoccupée par la mesure et l'objectivation, comme chez Georges Friedman (1955), et une "conception relativiste et conflictuelle" dans laquelle la qualification est enjeu de luttes et processus sociaux.

Le système taylorien favorise le développement d'une conception substantialiste de la qualification puisque, dans ce système, le travail étant prédéfini, le poste de travail et le travailleur qui l'occupe ne sont vus que comme réalisation d'une prescription. C'est la mise en évidence de l'écart existant entre travail "prescrit" et travail "réel" qui apporte les premières et sérieuses limites à cette approche.

La conception relativiste part de l'idée de P. Naville (1956) selon laquelle «La qualification c'est un jugement social sur la qualité des travaux » et affirme qu'il s'agit là "d'un processus de catégorisation constamment négocié", jugeant ce qui est qualifiable et ordonnant des catégories (Stroobants 1993a).

Dans la pratique, les processus de qualification mêlent constamment les deux registres. D'un côté, ils relèvent d'une négociation dans le cadre du rapport social entre employeurs et salariés. De l'autre, pour négocier, les partenaires ont besoin de repères collectifs qu'apportent les dispositifs d'objectivation et d'évaluation du travail.

Pour M. Stroobants (1993b), si "le salariat représente un dénominateur commun entre les travailleurs", la qualification est "un commun diviseur" servant à justifier les niveaux et les inégalités de rémunération.

Si les débats sur la qualification ont occupé une place de choix dans les réflexions de la sociologie française sur le travail et son évolution, celle-ci est restée longtemps aveugle et muette sur les divisions entre hommes et femmes à cet égard. Que l'approche "substantialiste", marquée par le déterminisme technologique, y ait été peu sensible peut se comprendre. Mais les approches "relativistes", où la qualification a pu être conçue comme "rapport social" (Maurice, 1986), n'ont pas été plus attentives aux différences entre hommes et femmes. 
Comment cette division sexuée des salariés au regard de la qualification s'est-elle peu à peu glissée dans l'analyse sociologique du travail ? Tel sera le fil conducteur de notre propos.

\section{La qualification des hommes et les qualités "naturelles" des femmes}

C'est la proximité entre les emplois occupés par les femmes et les tâches et rôles qu'elles remplissent dans le cadre de la famille qui apparaît tout d'abord aux yeux de certains auteurs ouvrant la voie à une réflexion sexuée sur la qualification.

Dans le foulée du mouvement féministe des années 60-70,un large débat s'instaure en France, après les Etats Unis, sur le travail domestique. Travail invisible, gratuit, "il traduit l'exploitation de la femme par l'homme" dans le cadre du "mode de production domestique" qui, pour C. Delphy (1998/1970), fonctionne parallèlement au "mode de production industriel". Elle montre que ce n'est pas la nature du produit ou du service qui en détermine le caractère marchand ou non mais le rapport social dans lequel il s'exerce, le statut sous lequel le travail est effectué (statut d'épouse ou de salariée) (Delphy, 1998/1978).

Mais C. Delphy et les sociologues féministes des années 70 , en se focalisant sur la construction théorique de la question de l'oppression féminine, laissent ouverte celle de la relation entre les deux "modes de production", domestique (patriarcat) et capitaliste, et dans le même temps, celle de la qualification.

A cet égard, l'étude antérieure de M. Guilbert (1966a) auprès d'ouvrières de la métallurgie parisienne apparaît pionnière. Faisant œuvre classique d'analyse des postes de travail, elle remarque que les caractéristiques de ceux-ci sont à rapprocher des caractéristiques opératoires des tâches ménagères : séquences courtes et répétitives, mouvements de faible amplitude, travaux sédentaires, postes isolés les uns des autres, faible responsabilité. Mais à cette époque, son travail "..(n'a) pas tellement attiré l'attention.....les collègues ne dépréciaient pas les gens qui s'occupaient du travail des femmes, mais ils considéraient que c'était plus un travail de femmes qu'un travail de sociologue." (Guilbert, 1999)

C'est D. Kergoat $(1978,1982)$ qui entame une vraie réflexion sexuée sur la qualification ouvrière. Elle constate que les employeurs apprécient et recherchent des qualités propres à la main d'œuvre féminine : habileté, dextérité, minutie, patience. Mais, contrairement à la force physique de l'ouvrier, ils ne les rétribuent pas. Ce ne sont pas pour eux des "savoir-faire", fruits d'une formation et susceptibles d'être "qualifiés" mais des "aptitudes" innées, liées à la nature féminine. Or pour l'auteure , "...au lieu de dire que les ouvrières ont une formation nulle et non adaptée, nous dirons au contraire qu'elles en ont une parfaitement adaptée aux emplois industriels qu'on leur propose, et qu'elles ont acquise d'abord par un apprentissage (au métier de future femme quand elles étaient petites filles) puis par une formation continue (travaux domestiques)" (Kergoat, 1982 : 66). Ainsi, chez les ouvrières, l'acquisition des savoir-faire se fait hors des canaux institutionnels et en référence à la sphère privée. Elle se fait donc individuellement contrairement aux ouvriers pour qui elle est collective. C'est pourquoi les ouvrières elles-mêmes intériorisent l'invisibilisation et la banalisation de leur propre qualification.

Ainsi, "apprentissage social par la petite fille de son rôle de future femme et déqualification féminine sont à analyser de pair" (Kergoat, 1978 : 77).

\section{Femmes et techniques}

D’une façon générale, dans toutes les sociétés, l'accès aux techniques -en particulier à celles considérées comme importantes pour la reproduction sociale dont le paradigme est 
représenté par les armes- constitue un des éléments et un enjeu de la domination sociale. Cela se vérifie aussi pour la domination masculine : la technique apparaît comme un enjeu des rapports de sexe. Paola Tabet (1998/1979) montre par exemple que, dans les sociétés de chasse et de ceuillette, les femmes sont exclues de la fabrication des outils et, bien sûr, des armes et que, d'une façon générale les outils qu 'elles emploient sont plus rudimentaires et moins spécialisés que ceux utilisés par les hommes.

D'un côté on aurait donc une constante des organisations sociales. De l'autre les sociétés modernes opèrent en fait une construction particulière du rapport sexué aux techniques. Ces sociétés, qui vont progressivement devenir « industrielles », sont fondées sur le pari d'une maîtrise voire d'un dépassement de la Nature. Or la technique, dans sa conception même, renvoie à l'idée de transformation de la nature ${ }^{1}$. Parallèlement, la Révolution française définit une nouvelle division sexuée de l'espace social : si le masculin est associé à la modernité de la Raison, du Travail, de la Liberté et du Citoyen, le féminin est lié à l'ordre social ancien de la famille, de la dépendance et de la naturalité ( Rosanvallon, 1992; Daune-Richard, 1997). Ainsi, dans les représentations sociales, l'homme est désormais vu comme porteur de la Culture - et in fine pouvant dominer la Nature- et la femme comme soumise à la Nature (Mathieu, 1991).

Aussi, au fur et à mesure que le travail s'instrumente, on assiste à une (re)définition des métiers qui, fondée sur l'articulation technique-métier-homme, exclut les femmes (Flament, 1985). La dépossession des sage-femmes au pofit des chirurgiens-accoucheurs, entre le $\mathrm{XVI}^{\circ}$ et le $\mathrm{XVIII}^{\circ}$ siècles, au motif que "les femmes ne doivent user d'aucun instrument » (Knibiehler et Fouquet, 1980, p.77) est exemplaire à cet égard. Au bout du compte, «la technique se constitue comme objet dans les rapports sociaux de sexe : elle y acquiert une "nature" masculine » et « ainsi constituée, la technique est à son tour constitutive des rapports de sexe : le Masculin y acquiert une nature "technique ".» (Flament, 1985, p.38).

Dans cette construction sociale, les techniques instrumentées sont associées aux métiers, donc au travail qualifié, et au masculin : technique, qualifié et masculin se conjuguent ensemble. On retrouve la force de cette association dans les conflits observés dans l'imprimerie, dans les années quatre-vingt (Maruani et Nicole, 1989) où, avec le passage de la composition " à chaud » à la composition « à froid » permise par l'informatisation, apparaît un nouveau métier, "claviste ", occupé par des femmes assimilées à des dactylos, qui marginalise progressivement les compositeurs, hommes, ouvriers du Livre ${ }^{2}$.

Ce qui apparaît le plus nouveau ici est que, dans les sociétés modernes, la division sexuée de l'accès aux techniques est fondée sur un rapport à la nature défini différemment au féminin et au masculin -soumission pour les femmes, maitrise pour les hommes- et non plus sur un rapport de pouvoir direct des hommes sur les femmes légitimé par un mythe des origines ou un ordre des dieux. C'est la définition du féminin comme lié à la nature qui fonde l'exclusion des femmes de la légitimité technique. Or l'un des clivages majeurs entre professions masculines et féminines repose sur la technicité reconnue du métier.

\section{Les services et le "relationnel" comme qualification}

La définition et la reconnaissance des qualifications dans les services pose des problèmes particuliers. Plus que les emplois industriels, les emplois de service mobilisent, à côté de compétences techniques, des compétences personnelles qui sont difficiles à mesurer. Si la

\footnotetext{
1 idée tout à fait contraire à ce qui prévaut dans les société antérieures où l'homme se représente comme soumis à la Nature en ce qu'elle est l'expression même de la création et de la volonté des dieux (pour une réflexion pionnière et approfondie à ce sujet, $\mathrm{cf}$ : Vernant, 1966, $4^{\circ}$ partie: Le travail et la pensée technique).

${ }^{2}$ A la même période, C. Cockburn (1983) fait une étude tout à fait comparable dans l'imprimerie britannique.
} 
maîtrise d'une technique, et donc l'efficacité de l'opérateur, peut être estimée par les quantités produites et la présence/absence de défaut dans le produit, la qualité du service met en jeu des capacités de contact, de communication, de diplomatie difficiles à évaluer -même si elles sont appréciées- parce qu'elles s'acquièrent plutôt par l'expérience et la socialisation que par des formations structurées. Or, dans la conception des dispositifs d'évaluation, les compétences qui ne passent pas par des procédures d'objectivation et de formalisation (comme, par exemple, la formation et le diplôme), ne sont a priori pas destinées à être "qualifiées", c'est à dire reconnues comme qualifications et monnayables, en tant que telles, dans les conventions collectives et les relations avec les employeurs.

Ces considérations s'appliquent particulièrement, on l'a vu, aux compétences considérées comme féminines : acquises dans la sphère privée de la famille et dans l'exercice des rôles domestiques féminins d'attention aux autres, elles sont perçues par les employeurs mais aussi, souvent, par les salariées elles-mêmes, comme des qualités "naturelles", attachées à leur identité personnelle et féminine et ne relevant pas -tout au moins pas immédiatement- du rapport salarial (Kergoat 1982 et 1992). Pourtant, des études ont montré que l'utilisation de ces compétences dans un travail rémunéré transformait profondément non seulement leurs conditions d'exercice mais aussi leurs caractéristiques intrinsèques (par exemple les règles d'hygiène et de sécurité ne sont pas les mêmes dans un cadre collectif -beaucoup plus normées- et dans le cadre d'un domicile privé) (Céreq, 1994 ; Labruyère, 1996).

On retrouve ici l'opposition entre le pôle des représentations sociales qui associe la technique et la maîtrise de la nature au masculin et celui qui conjugue le naturel et la soumission à la nature au féminin. Si la technicité, en tant qu'elle symbolise un pouvoir sur la nature, est associée au masculin et au travail qualifié, les services, définis par le relationnel, sont exclus d'une représentation en terme de technicité et sont vus comme appartenant à un univers de travail où sont sollicitées des qualités inhérentes à la nature féminine. Même lorsque la technicité d'un métier "féminin" s'accroît, elle tend à être disqualifiée par le sexe de l'opérateur. Un exemple connu est celui de la profession d'infirmière qui fait de plus en plus appel à des technologies nouvelles, tant dans le domaine strictement médical (matériel de diagnostic et de soins) que dans celui de la gestion administrative et des stocks, mais n'est pas pour autant associée à la représentation d'un métier technique. Les revendications actuelles de la profession concernent d'ailleurs le diplôme d'infirmière qui, délivré à l'issue d'une formation qui dure trois ans après le bac, est, dans les classifications, évalué à un niveau Bac +2 .

En fait, la représentation attachée à la profession d'infirmière est principalement associée aux tâches de soins ${ }^{3}$, mais dans leur aspect moins technique qu'humain. De plus, les fonctions d'infirmières ont été longtemps assurées, gratuitement, par des religieuses et l'association à l'idée de vocation est particulièrement forte. Or, qui dit vocation dit rapport immédiat -qui s'impose "naturellement" et individuellement- à la profession. Ainsi, dans leur mouvement de la fin des années 80 , avec leur mot d'ordre "ni bonnes, ni nonnes, ni connes", les infirmières cherchaient à être reconnues comme "professionnelles" et, ce faisant, à construire le relationnel comme qualification (Kergoat, 1992).

Il n'en demeure pas moins que les compétences mobilisées dans les services n'ont pas été suffisamment décortiquées. A. Soares (2002) montre que la dimension relationnelle des compétences, qui se référerait pour lui à la "capacité à garder l'équilibre de l'interaction", a souvent été utilisée comme une catégorie fourre-tout qu'il conviendrait de décomposer. Il propose en fait une analyse du travail dans les services à partir de trois dimensions. A côté de la dimension relationnelle, il identifie le "travail sexuel" : le corps et la sexualité étant utilisés par les employeurs comme une stratégie de marketing deviennent une partie constitutive de la

\footnotetext{
${ }^{3}$ Plus précisément ce que les anglophones englobent dans la notion de "care" et que M.-Th. Letablier (2001) a traduit par "le travail centré sur autrui"
} 
production des services. Enfin il insiste sur la dimension "émotionnelle" du travail dans les services et montre comment "à cause de la division sexuelle du travail émotionnel, non seulement les travailleuses ne voient pas la valorisation de leur travail mais elles sont en outre stigmatisées comme étant "émotives" voire hystériques." (p.246)

\section{La mobilité professionnelle}

La mobilité professionnelle est une voie d'accès à la qualification, or elle n'est pas du tout la même pour les hommes et pour les femmes.

Une étude récente de la mobilité en France (Chapoulie, 2000) montre la moindre ouverture des filières de mobilité féminines : si les hommes peuvent suivre une voie "technique" ou "administrative" ou encore celle d'une mise à son compte, seule la seconde est ouverte aux femmes. Enfin, si la mobilité descendante est rare chez les hommes, elle semble bien réelle chez les femmes et "dans les professions intermédiaires les chances de régression sont supérieures aux chances de promotion" (P. 41).

Ce modèle différencié de mobilité professionnelle en tant que voie d'accès à la qualification a été analysé par D. Kergoat. Dans les années 70, comparant la mobilité des ouvriers et celle des ouvrières dans un même bassin d'emploi, elle remarque que celles-ci se distinguent fortement tant dans leur nature que dans leur amplitude. Les hommes suivent des filières plus promotionnelles et plus diversifiées que les femmes. Les filières masculines sont industrielles (ouvrier non qualifié ->ouvrier qualifié -> éventuellement contremaître) ou tertiaires (ouvrier qualifié $->$ employé qualifié $->$ cadre tertiaire) ou conduisent à l'indépendance comme artisan ou petit commerçant. Pour les ouvrières, il s'agit plutôt d'une "mobilité horizontale, diffuse et contradictoire à tous les âges" (1982 : 95). Lorsqu'elles restent dans l'industrie, elles suivent une filière linéaire, du textile à l'électronique. Lorsqu'elles vont vers le tertiaire, elles deviennent le plus souvent (à près de $80 \%$ ) personnel de service ou employées qualifiées ou non qualifiées.

Enfin, ce qui distingue la mobilité des ouvrières de celle des ouvriers dans cette étude est la discontinuité professionnelle "qui se double le plus souvent d'une discontinuité au niveau de la qualification...A l'intérieur de la classe ouvrière, la paupérisation en fonction de l'âge est beaucoup plus intense pour les femmes que pour les hommes" (1982 : 99).

L'étroitesse de leur espace de mobilité professionnelle et l'imbrication du professionnel et du domestique conduit les femmes à des stratégies qu'on ne trouve pas chez les hommes. Il a ainsi été montré que le temps de travail devenait un enjeu de "promotion", partagé par des employeurs et leurs salariées, caissières et ouvrières (Bouffartigue et Pendariès, 1993 ; Pendariès, 1994). Chez les premières, à temps partiel, l'allongement de leur temps de travail "représente un enjeu essentiel : celui de gagner en maîtrise sur l'organisation temporelle de leur vie, tout en élevant un niveau de salaire contractuellement garanti" (1994 : 293). Chez les secondes, "le passage de l'équipe "du soir" à celle "du matin" constitue un enjeu suffisamment important, notamment pour les opératrices chargées de famille, pour que ce changement soit, là aussi, assimilé, par l'employeur comme par les ouvrières, à une "promotion"." (1994 : 294).

Mais la question de la mobilité professionnelle dans sa dimension verticale est profondément articulée à celle du pouvoir et de l'autorité.

L' accès des femmes aux fonctions hiérarchiques paraît limité par ce que les anglophones ont appelé un "plafond de verre", invisible donc. De fait, si, dans les denières décennies, elles sont de plus en plus nombreuses à être cadres, elles occupent alors beaucoup moins souvent que leurs collègues masculins des fonctions d'encadrement et d'organisation du travail (Volkoff, 1987) et sont surtout présentes dans des postes fonctionnels (administration, communication) ou d'expertise (Laufer, 1984 et 2001 ; Marry, 1992). En tout état de cause, 
elles sont particulièrement absentes des postes de décision : 6\%à 7\% seulement des cadres dirigeants des grandes entreprises françaises sont des femmes (Bertin-Mourot, 1997; Laufer et Fouquet, 2001) et $14 \%$ des emplois de la haute fonction publique sont occupés par des femmes (Martinez, 2001).

On rencontre ici une illustration de ce que les anthropologues, en particulier, ont mis au jour depuis longtemps : la division sociale des espaces du masculin et du féminin est asymétrique et hiérarchisée. Le masculin sert de référent général, il dit la vision légitime du monde, de l'ordre social et, ce faisant de l'ordre des sexes : il domine, matériellement et symboliquement ( Mathieu, 1991 ; Godelier, 1995 ; Bourdieu, 1998. Cf. aussi : DauneRichard et Hurtig, 1995).

De nombreux travaux ont montré que le monde du travail et de l'entreprise étaient profondément structurés par cet ordre des sexes. La raison et le pouvoir étant socialement associés au masculin, les organisations bureaucratiques, fondées sur la rationalité et la hiérachie, tendent à réserver aux femmes des places subordonnées (Laufer, 1984). Les processus par lesquels se met en place cette «sexuation» du pouvoir dans l'emploi sont multiples et complexes, passant y compris par la médiation de la sexualité (Aubert et alii, 1986). Dans les espaces mixtes du travail, où se côtoient hommes et femmes, on peut identifier un mécanisme quasi universel : la construction de la différence. Or on sait qu'en matière de catégorisation sociale, entre proclamer une différence et hiérarchiser, la voie est étroite (Guillaumin, 1982).

Des processus, souvent subtils et difficilement repérables, orientent les hommes et les femmes vers des parcours différents au sein de l'entreprise. En fait, même lorqu'ils occupent des postes équivalents, la nature des tâches effectuées n'est pas la même, les distinctions correspondant aux représentations de ce qui est masculin ou féminin. Ceci a été bien montré chez les employés, en particulier dans des milieux professionnels (fonction publique, sécurité sociale) où la définition des postes étant fortement codifiée, on s'attend à ce que les discriminations soient réduites ( Battagliola, 1984 ; Alonzo, 1996). Ces travaux montrent qu'il n'en est rien. Derrière les règles formelles s'inscrivent des pratiques informelles : d'un côté les hommes employés "jouent" -et mettent en avant- la différenciation avec les femmes occupant des postes en principe identiques ; de l'autre, les hiérarchies sollicitent et utilisent ces comportements différenciés pour orienter les hommes plus que les femmes vers des formations et/ou des postes plus prometteurs en terme de carrière. F.Battagliola (1984) montre ainsi que, dans une population d'employés de la sécurité sociale recrutés très jeunes, dans la même vague et avec les mêmes diplômes, dix ans plus tard, la moitié des hommes sont devenus cadres tandis que la majorité des femmes sont toujours dans des emplois d'exécution.

Les entreprises s'appuient, elles aussi, sur les représentations sociales du masculin et du féminin pour gérer différemment leur personnel selon le sexe. On le remarque dès l'entrée dans la vie active puisque le rapprochement des conditions d'insertion des jeunes diplômés n'a pas fait disparaître un déclassement relatif des jeunes femmes : quelques années après leur entrée dans la vie active, à diplôme égal, elles sont nettement moins souvent que leurs homologues masculins classées cadre ou profession intermédiaire (Couppié et alii, 1997; Galland et Rouault, 1996). Or, la "deuxième chance" que constitue la formation continue joue moins pour les femmes que pour les hommes. D'un côté, même si elles y accèdent de plus en plus, cette porte demeure plus ouverte aux hommes ; de l'autre, elles en tirent moins de bénéfices. Même lorsque, après une formation, elles connaissent un changement de poste ou de qualification, la reconnaissance salariale ne suit pas : ainsi, les écarts de salaires constatés entre hommes et femmes avant formation ne sont pas compensés mais accentués après (Croquey, 1995). On retrouve la difficulté, toujours plus grande pour une femme, de faire reconnaître ses compétences en termes de qualification, mais, plus encore, de prix et de salaire. 


\section{Qualifications et salaires : des enjeux de pouvoir}

Au total, les représentations sociales du masculin disent un ordre des sexes qui attribue au masculin les qualités socialement valorisées. Dans le monde du travail, cette opération passe par la qualification des emplois en tant «qu'estimation de leur valeur sociale » (Naville, 1955) et, in fine, par le prix qui leur est attribué. Car les systèmes de salaires sont des systèmes de signes qui, en utilisant le langage universel de la monnaie, disent la visibilité et l'invisibilité des compétences, leur légitimité et, au bout du compte, la vérité des acteurs et des rapports de force dans la négociation. Ce faisant, ils disent et redisent les ordres sociaux et, en particulier, l'ordre des sexes.

Ainsi, les compétences mobilisées dans les emplois "féminins" sont-elles occultées non seulement parce qu'elles sont "naturalisées" mais parce que le féminin s'efface devant le masculin. M. Godelier (1995) parle de processus "d'assimilation", "d'englobement" du féminin dans le masculin.

La hiérarchie sociale du masculin et du féminin fait disparaître les compétences exercées par les femmes des échelles d'évaluation du travail. La force physique, attribut masculin, est bien une compétence "naturelle", mais elle pèse d'un poids important dans cette évaluation. Ce n'est pas le cas des tâches pénibles de soins hospitaliers comme le nettoyage du sang, des vomissures et excréments, massivement effectuées par des femmes. Plus encore, la force physique mise en œuvre par les infirmières et aides soignantes dans le maniement des malades immobilisés est occultée et pas non plus considérée comme "qualifiable".

L'évaluation du travail et sa traduction en qualification et en prix est donc profondément marquée par les représentations sociales de l'ordre des sexes. Elles font jouer cette évaluation dans des sens différents pour les hommes et pour les femmes. Elles imprègnent les acteurs de la négociation -experts, employeurs et salariées elles-mêmes- et tendent à faire disparaître l'utilité même d'intégrer la dimension sexuée dans la négociation (Silvera, 1996 ; Lemière et Silvera, 1999). 
L'exemple des programmes d'équité salariale est particulièrement éclairant à cet égard (Chicha, 1997). C'est par l'évaluation et la réévaluation des rémunérations que se révèlent des compétences cachées, parfois aux yeux des femmes elles-mêmes, et qu'est mise en débat la question de la valeur sociale des compétences exercées ; on a ici une illustration du propos de M. Stroobants selon lequel le salaire est l'instrument, le médiateur des remaniements dans les définitions et les représentations de la qualification.

Mais ces réévaluations des compétences et des rémunérations exposent en même temps au grand jour les enjeux de hiérarchie et de pouvoir entre hommes et femmes, le problème pour les premiers étant de «maintenir l'écart» (Kergoat, 1992). Si, comme l'affirme M. Stroobants, la qualification est un "commun diviseur", alors les négociations autour de la qualification et des salaires révèlent les lignes de fractures dans les collectifs de travail. La domination masculine joue ici pleinement : la mise en concurrence des hommes et des femmes qui a marqué l'histoire syndicale (Guilbert, 1966b) en est une illustration. Le verrouillage de l'accès à la formation et, partant, au métier ${ }^{4}$, l'appropriation masculine de la sphère technologique ont construit la non-qualification des femmes et leur incompétence technique (Maruani et Nicole, 1989 ; Hirata et Rogerat, 1988). Mais en retour, le travail non qualifié et donc bon marché des femmes constitue une menace pour la qualification et les salaires masculins, en particulier dans les périodes de changements technologiques. Dans les négociations, les employeurs jouent alors la division hommes-femmes -ou, dit autrement, le rapport entre sexes sociaux-. Et les hommes ne sont pas toujours gagnants, même si l'avantage féminin s'avère souvent très relatif (Maruani et Nicole, 1989).

\section{Conclusion}

La sociologie du travail a eu bien du mal à voir et à analyser la qualification d'un point de vue sexué, et, en France, le travail de quelques pionnières n'est guère dépassé aujourd'hui.

En parcourant l'histoire de ce regard sexué, on remarque que, au regard de la littérature anglo-saxonne, la sociologie française a peu abordé la place des rapports de pouvoir directs des hommes sur les femmes dans l'accès à la qualification, en particulier par la médiation de la sexualité et de la sexuation des organisations (cf. par exemple : Cockburn, 1983 ; Hearn et alii, 1989 ; Mills et Tancred, 1992; Wacjman, 1998). De même, l'analyse des dispositifs d'évaluation du travail (job evaluation : cf. Steinberg, 1990 ; Horrel et alii, 1990) a peu été développée en France jusqu'aujourd'hui (cf. cependant : Rozenblatt et Sehili, 1999 et Séhili, 2000), probablement en lien avec la faible mobilisation des acteurs sociaux à cet égard (Lemière et Silvera, 1999).

On peut se demander si les débats autour de la substitution de la notion de compétence à celle de qualification (Dugué, 1994) et du développement d'un "modèle de la compétence" (Zarifian, 2001) mettront autant de temps à en étudier les implications sexuées. Pourtant, à cet égard, "ce sont les mêmes processus discriminants qui continuent...à jouer un rôle important dans la définition et l'évaluation des compétences..." (Séhili, 2000). De plus, l'ambiguïté qui entoure la composante "savoir être" dans la notion de compétence en favorise une acception naturaliste et se fait ainsi "l'instrument d'une légitimation rationnelle d'une forme aboutie de

\footnotetext{
${ }^{4}$ C.Omnès (1997) montre comment, dans la métallurgie parisienne du début du siècle, l'accès à la formation (CAP) par apprentissage était fermé aux jeunes femmes. La profession crée son propre système de qualification qui, "donnant une définition relativement ouverte de l'ouvrier professionnel dont la formation peut être acquise aussi bien par l'apprentissage que par la pratique, (...) confère une légitimité et une valorisation à la formation sur le tas donnée aux femmes." (p.45). Mais en 1936, la convention collective institue l'obligation du CAP pour accéder au grade d'ouvrier professionnel, alors même qu'il n'existe pas de filière d'apprentissage spécialisée et sanctionnée par un CAP pour les femmes : leur exclusion de la catégorie des ouvriers qualifiés est prononcée ( $\mathrm{p}$. 228).
} 
domination, celle qui amène les dominés à penser (et se penser) à travers les catégories de pensée des dominants..." (Sultzer, 1999).

En tout état de cause, un acquis des analyses "relativistes" et féministes de la qualification est que sa définition s'inscrit dans le jeu de rapports sociaux : rapport salarial et rapport entre les sexes, pour ce qui nous concerne ici. Mais c'est bien leur fonctionnement articulé -et non parallèle, tel que le voyait C. Delphy (1998/1978)- qui joue.

La qualification -et son prix : le salaire- est un enjeu central dans la dialectique croisée de ces deux rapports. Mais l'occultation de la dimension sexuée de l'enjeu apparait décisif en faisant disparaître certains termes de la négociation, voire l'utilité même de celle-ci.

En France, sur la longue durée du rapport salarial, les travailleurs ont développé des stratégies et mis en place des institutions dédiées à la négociation -même si celles-ci paraissent actuellement mises à mal par la logique compétence (Oiry et d'Iribarne, 2000). Jusqu'aujourd'hui, l'importance du rapport entre les sexes dans la gestion des qualifications n'a cependant pas débouché sur une réelle prise en compte de cet enjeu dans la négociation collective.

\section{Bibliographie}

Alonzo Philippe (1996), Femmes employées. La construction sociale sexuée du salariat, Paris, L'harmattan, Collection logiques sociales.

Aubert Nicole, Enriquez Eugène, de Gaulejac Vincent (1986), Le sexe du pouvoir. Femmes, hommes et pouvoir dans les organisations, Paris, Desclée de Brouwer.

Battagliola Françoise (1984), Employée et employées. Trajectoires professionnelles et familiales, in Le sexe du travail, Presses universitaires de Grenoble, p.57-70.

Bertin-Mourot Bénédicte (1997), La participation des femmes à l'exercice du pouvoir dans les grandes entreprises, Cahiers du Mage, 1, CNRS-IRESCO, Paris, p.37-50.

Bouffartigue Paul et Pendaries Jean-René (1993), Formes particulières d'emploi et gestion d'une main d'œuvre peu qualifiée. : le cas des caissières d'un hypermarché, Sociologie du travail, 3, p.337-359.

Bourdieu P., La domination masculine, Paris, Seuil, 1998

Chapoulie Simone (2000), Une nouvelle carte de la mobilité professionnelle, Economie et statistique, 331, p.25-45.

Campinos-Dubernet Myriam et Marry Catherine (1986), De l'utilisation d'un concept empirique : la qualification. Quel rapport à la formation, in Tanguy (ed.) : L'introuvable relation-emploi, Paris, La Documentation Française. P.197-232

Cereq - Mission nouvelles qualifications - mairie de Valbonne (1994), Professionnalité et compétences des ATSEM et des AS dans les écoles maternelles de la commune de Valbonne, ronéo, $22 \mathrm{p}$.

Chicha Marie-Thérèse (1997) :

- Programmes d'équité salariale et reconnaissance des qualifications : entre méthodologie et politique, Cahiers du Mage, 2, CNRS-IRESCO, Paris, p.13-19..

- L'équité salariale. Mise en oeuvre et enjeux, Cowansville, Québec, Les éditions Yvon Blais inc.

Cockburn Cynthia (1983), Brothers. Male Dominance and Technological Change, , Londres, Pluto Press.

Couppié Thomas, Epiphane Dominique et Fournier Christine (1997), Insertion professionnelle et début de carrière. Les inégalités de carrière résistent-elles au diplôme ? Cereq bref, 135. 
Crocquey E. (1995), La formation professionnelle continue : des inégalités d'accès et des effets sur la carrière peu importants à court terme, Travail et emploi, nº65, p.61-68.

Daune-Richard Anne-Marie (1997), Travail et citoyenneté : un enjeu sexué hier et aujourd'hui, in Bouffartigue P. et Eckert H. :Le travail à l'épreuve du salariat, Paris, L'harmattan, p.93-108 .

Daune-Richard Anne-Marie et Hurtig Marie-Claude (1995), Catégories et représentations de sexe : un débat loin d'être clos, in Ephesia, La place des femmes, Paris, La découverte, p.426-438.

Delphy Christine (1998), Economie politique du patriarcat, Paris, Syllepse :

- L'ennemi principal, p.31-56 (1 publication : 1970).

- Travail ménager ou travail domestique ? p.57-73 ( $1^{\circ}$ publication : 1978).

Demichel Francine (1995), Représentation et sujet de droit, in Ephesia, La place des femmes, Paris, La découverte, p.462-465.

Dugué Elizabeth (1994), La gestion des compétences : les savoirs dévalués, le pouvoir occulté, Sociologie du travail, 36 (3), p.273-292.

Flament Catherine (1985), Une question primitive et futuriste : "femmes et techniques", bief, 16, Centre d'Etudes Féminines de l'Université de Provence, p.25-40.

Fournier Christine (1997), Femmes des années 90 : diplôme, activité et emploi, in : femmes sur le marché du travail. L'autre relation formation-emploi, Cereq : Etudes $\mathrm{n}^{\circ} 70$, p.47-60.

Friedmann, Georges (1955), Quelques aspects récents de l'éclatement des tâches industrielles, Journal de psychologie normale et pathologique, 1, p.77-96.

Galland O. et Rouault D., Des études supérieures inégalement rentables selon les milieux sociaux, INSEE Première, 469,1996

Godelier Maurice (1995), Du quadruple rapport entre les catégories de masculin et de féminin, in Ephesia, La place des femmes, Paris, La découverte, p.439-442.

Guibert Madeleine :

- (1966a), Les fonctions des femmes dans l'industrie, Paris, Mouton.

- (1966b), Les femmes dans l'organisation syndicale avant la guerre de 1914, Paris, Ed. du CNRS. 20.

- (1999), entretien avec M. Maruani et Ch. Rogerat, Travail, genre et société, 1, avril, p.7-

Guillaumin Colette (1982), Le choux et le moteur à deux temps. De la catégorie à la hiérarchie, Le genre humain, 2, p.20-36.

Hearn Jeff, Sheppard Deborah 1., Tancred-Sheriff Peta, Burrel Gibson (1989), The sexuality of organization, Londres, Sage

Hirata Helena et Rogerat Chantal (1988), Technologie, qualification et division sexuelle du travail, Revue française de sociologie, XXIX, p. 171-192.

Horrell Sara, Rubery Jill, Burchell Brendan (1990), Gender and skills, Work, Employment and Society, vol. 4, n², p.189-216.

Kergoat Danièle :

- (1978), Ouvriers=ouvrières? Propositions pour une articulation théorique de deux variables : sexe et classe sociale, Critiques de l'économie politique, nouvelle série, $\mathrm{n}^{\circ} 5$, p.6597.

- (1982), Les ouvrières, Paris, Syros.

Kergoat Danièle, avec la collaboration de F Imbert (1992), Ouvrières et infirmières : deux rapports à la qualification, in Kergoat D., Imbert F., Le doaré H., Sénotier D., Les ouvrières et leur coordination. 1988-1989, Paris, Lamarre, p.56-66.

Knibiehler Yvonne et Fouquet Catherine (1980), Histoire des mères du Moyen Age à nos jours, Paris, Montalba. 
Labruyere Chantal (1996), Professionnaliser les emplois familiaux, un objectif affirmé mais un processus encore à construire, Cereq bref, 125.

Laufer Jacqueline (1984), Les femmes cadres dans l'organisation, Le sexe du travail, Presses Universitaires de Grenoble, p.71-96.

Laufer Jacqueline et Fouquet Annie (2001), A l'épreuve de la féminisation in Cadres : la grande rupture, sous la direction de P. Bouffartigue. Paris, La Découverte p. 249-268.

Lemière Séverine et Silvera Rachel (1999), Comparaison de la valeur du travail et évaluation de l'emploi en vue de l'égalité salariale entre hommes et femmes : étude de faisabilité. Matisse. Etude ${ }^{\circ}{ }^{4} 7$, réalisée pour le Service des droits des femmes, mars.

Letablier Marie-Thérèse (2001), Le travail centré sur autrui et sa conceptualisation en Europe, Travail, genre et société, 6, octobre, p.19-41.

Marry Catherine (1992), Femme et ingénieur : la fin d'une incompatibilité ? La recherche, 241, volume 23, p.362-363.

Martinez Roger (2001), Les femmes dans l'encadrement supérieur de l'Etat au $1^{\circ}$ juin 2000, Point Stat, 01.01. 4 p.

Maruani Margaret et Nicole Chantal (1989), Au labeur des dames, Paris, Syros.

Mathieu Nicole-Claude (1991), L'anatomie politique, Paris, Côté femmes :

- Notes pour une définition sociologique des catégories de sexe, p.17-41 (1ère édition 1971).

- Homme-culture et femme-nature, p.43-61 (1ère édition 1973).

Maurice Marc (1986), La qualification comme rapport social : à propos de la "qualification" comme "mise en forme" du travail, in Salais et Thévenot (eds), Le travail. Marché, règles, conventions, Paris, Insee/Economica, p.179-192.

Mills Albert J. and Tancred Peta (1992), Gendering Organizational Analysis, Londres, Sage..

Naville Pierre (1955), Essai sur la qualification, Paris, Librairie Marcel Rivière.

Oiry Ewan et d'Iribarne Alain (2000), La notion de compétence : continuités et changements par rapport à la notion de qualification, Sociologie du travail, 43, p.49-66.

Omnès, Catherine (1997), Ouvrières parisiennes. Marché du travail et trajectoires professionnelles au $20^{\circ}$ siècle, Paris, Ed. de l'EHESS.

Pendaries Jean-René (1994), "Qualités féminines", temps de travail et qualification : les caissières d'un hypermarché et les ouvrières d'une usine de construction électrique, in Lucas Yvette et Dubar Claude (éds), Genèses et dynamiques des groupes professionnels, Lille, PUL, p. 287-298.

Rosanvallon Pierre (1992), Le sacre du citoyen, Paris, Gallimard.

Rozenblatt Patrick et Séhili Djaouida (1999), Chronique d'une neutralité annoncée, Formation - emploi, 68, p.21-36.

Séhili Djaouida (2000), De la "qualification" à la "compétence" : du changement pour les femmes?, in Le mirage de la compétence, Paris, Syllepse. P. 139-152.

Silvera Rachel (1996), Le salaire des femmes : toutes choses inégales par ailleurs...Paris, La Documentation Française.

Soarès Angelo (2002), Le prix d'un sourire. Travail, émotion et santé dans les services; in Harrisson D. et Legendre C., Santé, sécurité et transformation du travail. Presses de l'Université du Québec. P. 230-250.

Steinberg Ronnie J. (1990), Social Construction of Skill : Gender, Power and Comparable Worth, Work And Occupations, vol. 17, n4, p.449-479.

Stroobants Marcelle (1993),

- Savoir-faire et compétences au travail, Bruxelles éditions de l'université,.

- Sociologie du travail, Nathan, 128. 
Sultzer Emmanuel (1999), Objectiver les compétences sociales d'interaction. Critique sociale du savoir être, Education permanente, 140, p.51-59.

Tabet Paola (1998), Les mains, les outils, les armes, in . Tabet P., La construction sociale de l'inégalité des sexes. Des outils et des corps, Paris, L'Harmattan, Bibliothèque du féminisme, p. 9-20, . (1ère publication : 1979).

Vernant Jean-Pierre (1966), Mythe et pensée chez les Grecs, Paris, Maspéro.

Volkoff Serge (1987), L'encadrement : de la catégorie statistique à la fonction exercée, Economie et statistique, 204, p.29-33.

Wajcman Judy (1998), Managing Like a Man : Women and Men in Corporate Management, Londres, Polity Press.

Zarifian Philippe (2001), Le modèle de la compétence, Paris, Liaisons. 